\title{
Erdheim-Chester disease mimicking an intracranial trigeminal schwannoma: case report
}

\author{
Maysam Alimohamadi, MD, ${ }^{1,2}$ Christian Hartmann, MD, PhD, ${ }^{3}$ Vincenzo Paterno, MD, ${ }^{1}$ and \\ Madjid Samii, MD, PhD',2

\begin{abstract}
IInternational Neuroscience Institute; ${ }^{3}$ Department of Pathology, Medical School of Hannover, Germany; and 2 ranian International Neuroscience Institute, Tehran University of Medical Sciences, Tehran, Iran
\end{abstract}

\begin{abstract}
Erdheim-Chester disease (ECD) is non-Langerhans histiocytosis that can affect multiple organ systems. It usually affects middle-aged patients, and only a few reports of ECD in children appear in the literature. Central nervous system involvement is a common feature that usually occurs as infiltration of the hypothalamus-pituitary axis, cerebellum, and/ or brainstem. Meningeal involvement occurs less commonly. In this article, the authors discuss a rare pediatric case of ECD presenting as an infiltrative mass of the trigeminal nerve and resembling the clinical and imaging features of a trigeminal schwannoma.
\end{abstract}

http://thejns.org/doi/abs/10.3171/2014.10.PEDS14441

KEY WORDS brain tumor; Erdheim-Chester disease; histiocytosis; trigeminal schwannoma; skull base tumor; oncology

$\mathrm{E}$ RDHEIM-Chester disease (ECD) is a rare, sporadic non-Langerhans histiocytosis with unknown etiology. It typically affects middle-aged patients and has a significant male predominance. ${ }^{3,5,8,14,17,18,23,27,28,32}$ Only 8 pediatric cases have ever been reported. . $^{17,20,29}$

This disease is known to involve multiple organ systems, including the skeletal, cutaneous, cardiovascular, respiratory, urinary, and central nervous systems. Neurological involvement has been reported in up to $50 \%$ of patients. ${ }^{14,17,20}$ Involvement of the neuraxis occurs in both intraaxial and extraaxial compartments. ${ }^{14}$ The most common clinical form of neurological involvement is neuroendocrine dysfunction due to infiltration of the hypothalamus-pituitary axis; diabetes insipidus has been reported in approximately $25 \%$ of patients. ${ }^{14,15,18}$ Other frequent neurological presentations are cerebellar and pyramidal syndromes. ${ }^{18}$

In this article we describe a novel presentation of ECD in a child and review the most recently published literature about neurological involvement in this rare condition.

\section{Case Report}

History and Examination

A 14-year-old boy was evaluated for hypesthesia and dysesthesia of the first and third branches of the right trigeminal nerve and diplopia on looking left and up. Two years earlier, he had trigeminal neuralgia and diplopia for 1 week that resolved after medical therapy. Brain CT scanning with bone windows revealed scalloping of the right petrous apex due to an expansive mass of the right Meckel's cave and relative widening of the right foramen ovale (Fig. 1A and B). Contrast-enhanced brain MRI showed a homogeneously Gd-enhancing mass in the right cavernous sinus and Meckel's cave, extending to the posterior fossa through an enlarged trigeminal porus and extending to the infratemporal fossa through the foramen ovale (Fig. 1C and D).

\section{Operation}

The patient underwent surgery with a preoperative di-

ABBREVIATIONS ECD = Erdheim-Chester disease; JXG = juvenile xanthogranuloma; $L C H$ = Langerhans cell histiocytosis; RDD = Rosai-Dorfman disease. SUBMITTED August 19, 2014. ACCEPTED October 17, 2014.

INCLUDE WHEN CITING Published online February 27, 2015; DOI: 10.3171/2014.10.PEDS14441.

DISCLOSURE The authors report no conflict of interest concerning the materials or methods used in this study or the findings specified in this paper. 

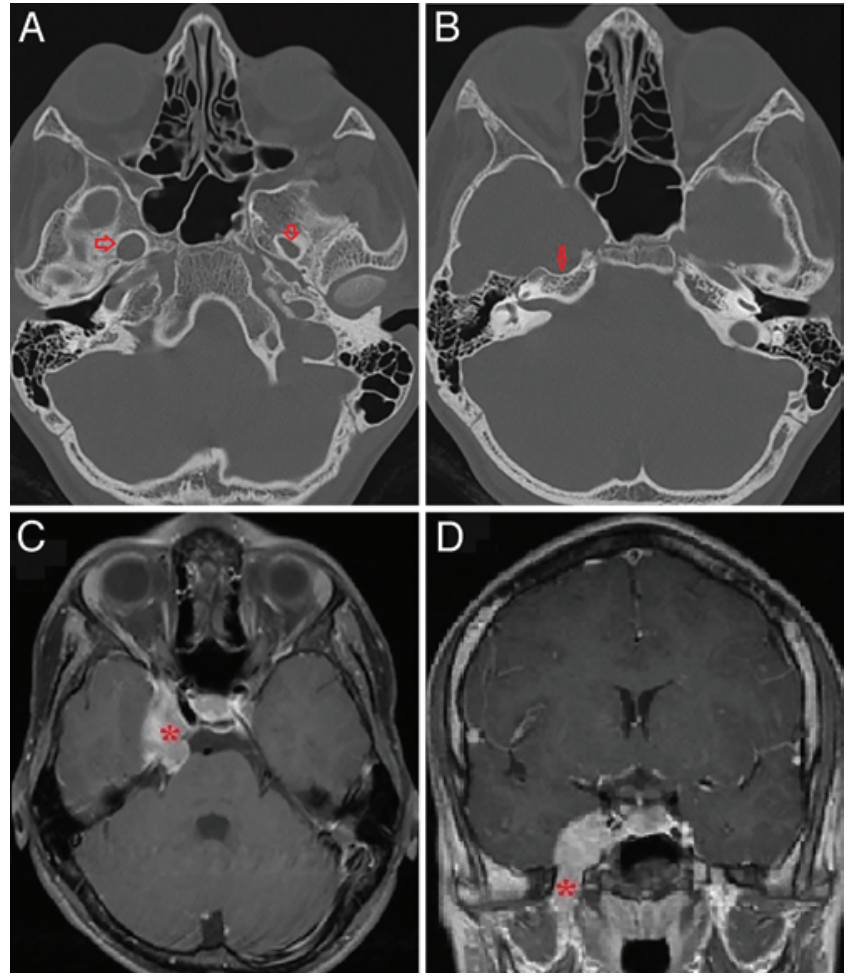

FIG. 1. A: Axial CT scan of the skull base showing widening of the right foramen ovale (horizontal arrow) compared to the left one (vertical arrow). B: Scalloping of the right petrous apex (vertical arrow). C and D: Axial and coronal postcontrast brain MR images showing a Gd-enhancing mass (asterisks) in the middle fossa with a small extension into the posterior fossa through the porus trigeminus and with extracranial extension through the foramen ovale. These imaging features are typically found in trigeminal nerve schwannomas. Figure is available in color online only.

agnosis of trigeminal schwannoma. Using a 3D neuronavigation system and continuous neurophysiological monitoring, we performed a linear skin incision and temporal craniotomy on the right side. The temporal lobe was extradurally retracted, and the middle fossa floor was drilled flush with Meckel's cave and the foramen ovale. The lateral wall of the foramen ovale was drilled away, and the dural sleeve of the gasserian ganglion and mandibular nerve was opened. The lesion had no distinct capsule and was diffusely infiltrating the mandibular nerve fibers (Fig. 2). Intraoperative frozen section revealed an inflammatory histiocytic lesion, so microsurgical subtotal decompression of the lesion was performed with preservation of the unaffected trigeminal nerve fibers. The dural opening was closed, and the subtemporal cavity was packed with Gelfoam.

\section{Histopathological Findings}

The histopathological evaluation (Fig. 3) revealed a peripheral nerve that was nearly completely destroyed by an inflammatory reaction with focal fibrosis. The infiltrating cells predominately consisted of histiocytes with a foamy cytoplasm. The histiocytes demonstrated large vesicular nuclei and occasionally prominent nucleoli. Furthermore, a strong lymphocytic component was seen. Only very rarely did eosinophilic granulocytes appear, and no signs of emperipolesis were observed. On immunohistochemical analysis, the histiocytes were positive for CD68. Only a few cells expressed S100. Not a single cell was found to be positive for CD1a. The majority of lymphocytes expressed the T-cell marker CD3, and only a minor fraction expressed the B-cell marker CD20.

\section{Postoperative Course}

The patient's postoperative course was uneventful except for increased hypesthesia in the area of the mandibular branch of the right trigeminal nerve. He was referred to a pediatric oncologist for evaluation of the systemic involvements, and he received adjuvant chemotherapy. $\mathrm{He}$ had typical skeletal involvements (as stated below), but there was no cardiovascular or internal organ involvement. He has received cytotoxic and corticosteroid therapy and has been under the care of a pediatric oncologist for 8 years now.

\section{Discussion}

Erdheim-Chester disease was first described as "lipoid granulomatose" by Jakob Erdheim and William Chester in 1930. ${ }^{12}$ Since then, 500-550 cases have been reported up until May 2014..$^{14}$ Most described patients have been between 40 and 70 years old, and a large series of 53 patients had a mean age of 55 years. ${ }^{14,17,18}$ Almost all studies have reported a strong male predominance, which also occurs in the above-mentioned series (75\%). ${ }^{18}$ By 2014 , only 8 pediatric cases were reported in the medical literature. ${ }^{17,20,29}$

For a long time, it remained unclear whether ECD was the histological manifestation of some kind of abnormal immune response or whether it was a neoplastic lesion. ${ }^{31}$ The recent discovery of $B R A F^{V 600 E}$ mutations only in the histiocytic cell component in approximately $50 \%$ of analyzed cases now clearly indicates that ECD is indeed a mutation-driven clonal neoplastic lesion and not a lesion due to aberrant immunological stimulation. ${ }^{19}$

\section{Histopathological Findings}

The definitive diagnosis of ECD is based on typical histopathological findings in an appropriate clinical and radiological context. ${ }^{14,27}$ The most frequent biopsy sites are sclerotic bone lesions and retro-orbital masses. ${ }^{27}$ The typical pattern is a polymorphic granuloma composed of lipid-laden (foamy or spumous) histiocytes and fibrosis or xanthogranulomatosis. ${ }^{16-18,30}$ The characteristic multinucleated Touton-type giant cells are often present. On immunohistochemical staining, the histiocytes are uniformly positive for CD68, negative for CD1a, and only rarely positive for S100 (negative for S100 in 80\% of cases). . $^{14,18,27,32}$

The Langerhans cells seen in Langerhans cell histiocytosis $(\mathrm{LCH})$ are differentiated from those seen in ECD by their immunohistochemical features: they are positive for CD1a and S100.14,32 Rosai-Dorfman disease (RDD) is another type of non-Langerhans histiocytosis which may also involve the CNS. The lesions of RDD consist of histiocytic proliferation exhibiting emperipolesis coupled with characteristic cytoplasmic staining against S100 protein. ${ }^{27}$ 

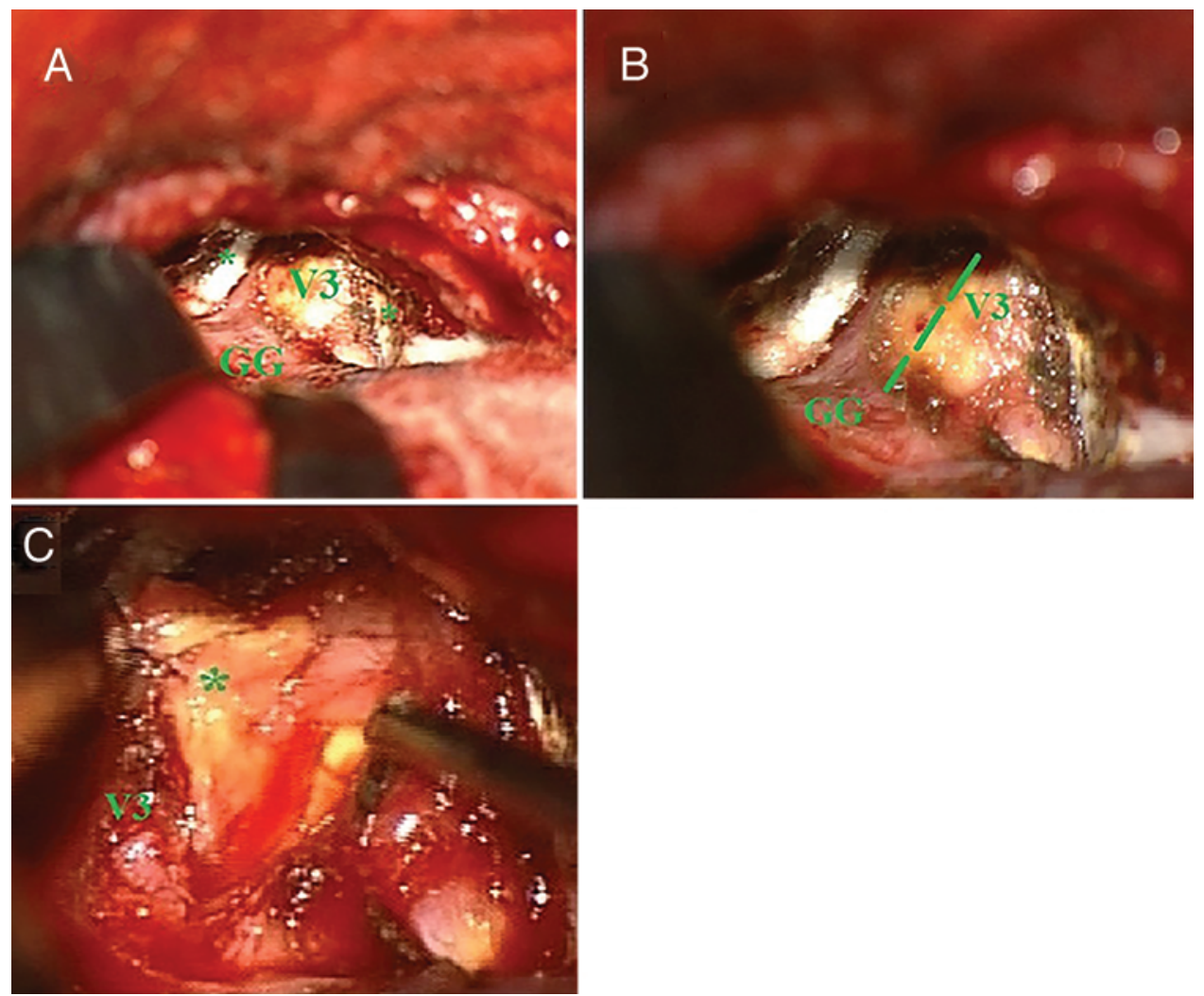

FIG. 2. A: Exposure of the gasserian ganglion (GG) and the mandibular division of the trigeminal nerve (V3) after lateral margins of the foramen ovale (asterisks) were drilled away. B: High-magnification view of the V3 passing through a dilated foramen ovale. The dashed line indicates the incision of the dural sleeve of the V3. C: The lesion is exposed without any definable capsule, infiltrating the V3 nerve fibers. Figure is available in color online only.

\section{Radiological Findings}

In addition to the histological features, the appearance of symmetric diaphyseal and metaphyseal sclerotic lesions of the legs is a nearly universal feature on radiography, with increased labeling on ${ }^{99} \mathrm{Tc}$ bone scanning. 1,3,5,8,11,14,16-20,23,27-32 Erdheim-Chester disease is a multisystemic disease and can involve almost any organ system. Patients with ECD frequently have CNS involvement as an extraskeletal manifestation. The frequency of CNS involvement in ECD varies from $25 \%$ to $50 \%$ of cases. ${ }^{11,14}$ Based on MRI findings, two patterns of abnormality have been described $\left.{ }^{8,17}: 1\right)$ a meningeal pattern that appears as meningioma-like mass lesions or nodular thickening of the dura mater, and 2) the more common infiltrative pattern that characteristically demonstrates hyperintense areas on T2-weighted and FLAIR sequences, with enhancing lesions frequently involving the cerebellum, brainstem, and periventricular white matter. ${ }^{8,23}$ Spotted areas of intense enhancement after Gd injection are usually present. , $^{87,32}$ A characteristic MRI feature is the prolonged retention of Gd by the lesions even after several days. ${ }^{22,24,27,32}$

Hypothalamus-pituitary axis involvement may be seen as a single micronodular or nodular infundibular stalk mass or as thickening of the pituitary stalk..$^{21,28} \mathrm{~A}$ sellar mass with extension to the cavernous sinus has been reported much less commonly.

Retro-orbital soft tissue infiltration and exophthalmos is seen in $25 \%$ of the patients. ${ }^{14}$ Spinal intradural and extradural masses have also been reported in ECD.,2,410

Infiltrative CNS lesions in LCH and ECD are similar in distribution and appearance, except for 3 features: the meningeal lesions of ECD are more expansive and voluminous, the spinal cord is spared in $\mathrm{LCH}$, and a degenerative atrophic process of the posterior fossa is occasionally described for LCH but never for ECD. ${ }^{2,4,10}$

\section{Clinical Symptoms}

The disease produces protean clinical manifestations as a result of the involvement of multiple organ systems. It can vary from asymptomatic or minimally symptomatic bone lesions to a severe multisystem disease that significantly shortens the patient lifespan. ${ }^{32}$ Although neurological symptoms have been described as the first clinical manifestation of ECD in less than one-third of cases, the disease is not diagnosed in most patients until the onset of such symptoms. ${ }^{8,17}$ The precise incidence of neurological involvement remains unclear as there is a bias toward reporting cases with unusual manifestations..$^{32}$ Extraaxial lesions present with focal symptoms due to local compression or with cognition and gait deterioration when the lesions are diffuse and bulky. ${ }^{14}$ Parenchymal infiltrations are responsible for severe functional handicaps in almost all patients. ${ }^{14,17}$ The most common neurological manifestations of ECD are diabetes insipidus and cerebellar and 


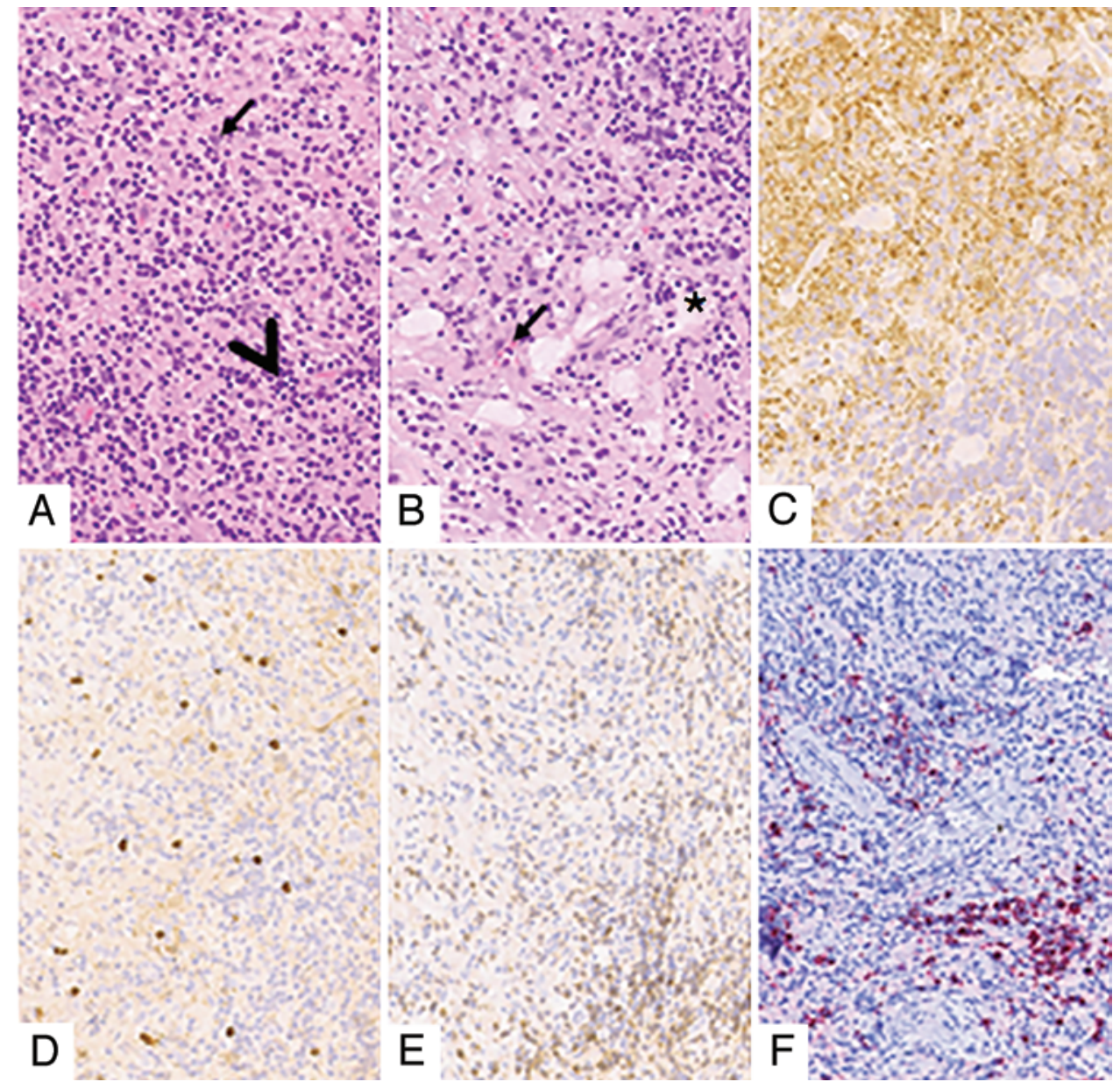

FIG. 3. Dense inflammatory reaction consisting predominantly of histiocytes (A, arrow) and lymphocytes (A, arrowhead). The histiocytes partially exhibited a foamy cytoplasm (B, arrow), and only very rarely did eosinophilic granulocytes appear (B, star). The histiocytes were positively labeled with CD68-specific antibodies (C), and only a few cells expressed S100 (D). Most of the lymphocytes demonstrated a CD3-positive T-lymphocytic (E) and, to a lesser extent, a CD20-positive B-lymphocytic (F) immunophenotype. Figure is available in color online only.

brainstem syndromes. ${ }^{3,14,17,18,27}$ Cerebellar symptoms are often attributable to involvement of the dentate and peridentate regions and the superior cerebellar peduncle and include ataxia, nystagmus, and gait disturbance. ${ }^{15,27}$ Other less common neurological features described include seizures, headaches, neuropsychiatric manifestations, sensory disturbances, cranial nerve paresis, and asymptomatic lesions. ${ }^{19}$ In a review of 33 patients with ECD, only 3 had normal brain imaging and most had lesions at 2 or more anatomical sites..$^{15}$ The systematic use of MRI has been recommended to investigate the brain in all, even asymptomatic, patients with ECD. ${ }^{18}$

\section{Differential Diagnosis}

In the absence of typical extracranial clinical manifestations, the diagnosis of ECD is one of exclusion. Other histiocytic disorders that should be considered in the differential diagnosis are neurosarcoidosis, $\mathrm{LCH}$, and RDD. Although many clinical clues can help to distinguish these conditions from ECD, definite differentiation is only possible via histopathological examination.

Juvenile xanthogranuloma (JXG) can cause similar in- tracranial lesions that are histopathologically identical to those in ECD. ${ }^{14,27}$ Given the clinically and histopathologically similar skeletal and retroperitoneal lesions in these two conditions, it has been suggested that ECD is a variant of JXG with predominantly noncutaneous involvement.., 14 Nevertheless, JXG is less commonly a multisystem disease than ECD. ${ }^{11}$

Other important differential diagnoses of intracranial ECD include demyelinating diseases, in particular multiple sclerosis; ${ }^{27,32}$ granulomatous infectious diseases such as tuberculosis, toxoplasmosis, and fungal infections; central pontine myelinolysis; and posterior reversible encephalopathy syndrome. ${ }^{8}$

\section{Treatment}

Currently, there are no universally accepted treatments for ECD. In general, initiation of therapy, rather than observation, is recommended for all symptomatic patients. A detailed discussion of the different therapeutic options is beyond the scope of this article. Briefly, however, the treatments described for ECD include corticosteroids, chemotherapy, radiotherapy, and immunotherapy. Radiotherapy 
for ECD usually yields no response or, at best, short-term palliation with disease progression within months. ${ }^{25,26}$ The reported adjuvant therapies for intracranial histiocytic disorders have led to only temporary relief of symptoms in ECD. ${ }^{3}$ In 2005, it was shown that interferon- $\alpha 2 a$ is the best initial treatment for ECD. ${ }^{14,18}$ In 2010, an activating mutation of the proto-oncogene $B R A F$ was shown to be involved in the pathogenesis of ECD. ${ }^{7}$ Treatment with inhibitors of $B R A F$ activation has shown promising results as an alternative to interferon therapy for ECD patients carrying such a mutation. ${ }^{14,18}$ A paucity of pediatric cases of ECD means that evidence regarding treatment is limited to case reports. ${ }^{14}$ The role of surgical debulking in ECD is limited to severe orbital lesions or surgically resectable intracranial lesions..$^{14}$

\section{Prognosis and Surveillance}

The biological behavior of systemic ECD is variable..$^{13,27}$ The extent and distribution of the disease determine the clinical course. ${ }^{18}$ The presence of widespread systemic disease appears to be an ominous prognostic feature. ${ }^{3,27}$ A multicenter survival analysis of 53 patients with CNS involvement by ECD revealed that such involvement was the sole independent clinical predictor of a fatal outcome. ${ }^{5}$ Fluorodeoxyglucose positron emission tomography scanning allows detection of CNS involvement and reveals early responses of CNS lesions to therapy. ${ }^{6}$ The performance of such imaging studies every 3-6 months is suggested for all patients following initiation of treatment. ${ }^{14}$

According to our review of the literature, this case is the 9th instance of ECD in children and the first instance of ECD presenting as a mass in Meckel's cave and the cavernous sinus with extracranial extension through the foramen ovale. Erdheim-Chester disease can mimic many types of parenchymal and skull base tumors and should be considered in the differential diagnosis of frozen section analysis for any skull base lesion containing multinucleated giant cells.

\section{References}

1. Adle-Biassette H, Chetritt J, Bergemer-Fouquet AM, Wechsler J, Mussini JM, Gray F: Pathology of the central nervous system in Chester-Erdheim disease: report of three cases. J Neuropathol Exp Neurol 56:1207-1216, 1997

2. Albayram S, Kizilkilic O, Zulfikar Z, Islak C, Kocer N: Spinal dural involvement in Erdheim-Chester disease: MRI findings. Neuroradiology 44:1004-1007, 2002

3. Alfieri A, Gazzeri R, Galarza M, Neroni M: Surgical treatment of intracranial Erdheim-Chester disease. J Clin Neurosci 17:1489-1492, 2010

4. Al-Quran S, Reith J, Bradley J, Rimsza L: Erdheim-Chester disease: case report, PCR-based analysis of clonality, and review of literature. Mod Pathol 15:666-672, 2002

5. Arnaud L, Hervier B, Néel A, Hamidou MA, Kahn JE, Wechsler B, et al: CNS involvement and treatment with interferon- $\alpha$ are independent prognostic factors in ErdheimChester disease: a multicenter survival analysis of 53 patients. Blood 117:2778-2782, 2011

6. Arnaud L, Malek Z, Archambaud F, Kas A, Toledano D, Drier A, et al: 18F-fluorodeoxyglucose-positron emission tomography scanning is more useful in followup than in the initial assessment of patients with Erdheim-Chester disease. Arthritis Rheum 60:3128-3138, 2009
7. Badalian-Very G, Vergilio JA, Degar BA, MacConaill LE, Brandner B, Calicchio ML, et al: Recurrent BRAF mutations in Langerhans cell histiocytosis. Blood 116:1919-1923, 2010

8. Bianco F, Iacovelli E, Tinelli E, Locuratolo N, Pauri F, Fattapposta F: Characteristic brain MRI appearance of Erdheim-Chester disease. Neurology 73:2120-2122, 2009

9. Breuil V, Brocq O, Pellegrino C, Grimaud A, Euller-Ziegler L: Erdheim-Chester disease: typical radiological bone features for a rare xanthogranulomatosis. Ann Rheum Dis 61:199-200, 2002

10. Caparros-Lefebvre D, Pruvo JP, Rémy M, Wallaert B, Petit $\mathrm{H}$ : Neuroradiologic aspects of Chester-Erdheim disease. AJNR Am J Neuroradiol 16:735-740, 1995

11. Caputo R, Marzano AV, Passoni E, Berti E: Unusual variants of non-Langerhans cell histiocytoses. J Am Acad Dermatol 57:1031-1045, 2007

12. Chester W: Über lipoidgranulomatose. Virchows Arch 279: 561-602, 1930

13. Chetritt J, Paradis V, Dargere D, Adle-Biassette H, Maurage CA, Mussini JM, et al: Chester-Erdheim disease: a neoplastic disorder. Hum Pathol 30:1093-1096, 1999

14. Diamond EL, Dagna L, Hymna DM, Cavalli G, Janku F, Estrada-Veras J: Consensus guidelines for the diagnosis and clinical management of Erdheim-Chester disease. Blood 124:483-492, 2014

15. Drier A, Haroche J, Savatovsky J, Godenèche G, Dormont D, Chiras J, et al: Cerebral, facial, and orbital involvement in Erdheim-Chester disease: CT and MR imaging findings. Radiology 255:586-594, 2010

16. Haroche J, Amoura Z, Dion E, Wechsler B, CostedoatChalumeau N, Cacoub P, et al: Cardiovascular involvement, an overlooked feature of Erdheim-Chester disease: report of 6 new cases and a literature review. Medicine (Baltimore) 83:371-392, 2004

17. Haroche J, Arnaud L, Amoura Z: Erdheim-Chester disease. Curr Opin Rheumatol 24:53-59, 2012

18. Haroche J, Arnaud L, Cohen-Aubart F, Hervier B, Charlotte F, Emile JF, et al: Erdheim-Chester disease. Curr Rheumatol Rep 16:412, 2014

19. Haroche J, Charlotte F, Arnaud L, von Deimling A, HéliasRodzewicz Z, Hervier B, et al: High prevalence of BRAF V600E mutations in Erdheim-Chester disease but not in other non-Langerhans cell histiocytoses. Blood 120:2700-2703, 2012

20. Jeon IS, Lee SS, Lee MK: Chemotherapy and interferonalpha treatment of Erdheim-Chester disease. Pediatr Blood Cancer 55:745-747, 2010

21. Johnson MD, Aulino JP, Jagasia M, Mawn LA: Erdheimchester disease mimicking multiple meningiomas syndrome. AJNR Am J Neuroradiol 25:134-137, 2004

22. Kujat C, Junk B, Hermes M, Martin J, Dewes W: [Cerebral manifestations of Erdheim-Chester disease.] Radiologe 31:307-309, 1991 (Ger)

23. Liotta EM, Jhaveri MD, Fox JC, Parameswaran V, Lewis SL: Erdheim-Chester disease. Arch Neurol 69:1514-1515, 2012

24. Martinez R: Erdheim-Chester disease: MR of intraaxial and extraaxial brain stem lesions. AJNR Am J Neuroradiol 16:1787-1790, 1995

25. Mascalchi M, Nencini P, Nistri M, Sarti C, Santoni R: Failure of radiation therapy for brain involvement in Erdheim Chester disease. J Neurooncol 59:169-172, 2002

26. Miller RC, Villà S, Kamer S, Pasquier D, Poortmans P, Micke O, et al: Palliative treatment of Erdheim-Chester disease with radiotherapy: a Rare Cancer Network study. Radiother Oncol 80:323-326, 2006

27. Rushing EJ, Bouffard JP, Neal CJ, Koeller K, Martin J, Ozdemirli M, et al: Erdheim-Chester disease mimicking a primary brain tumor. Case report. J Neurosurg 100:1115-1118, 2004 
28. Sedrak P, Ketonen L, Hou P, Guha-Thakurta N, Williams MD, Kurzrock R, et al: Erdheim-Chester disease of the central nervous system: new manifestations of a rare disease.

AJNR Am J Neuroradiol 32:2126-2131, 2011

29. Tran TA, Fabre M, Pariente D, Craiu I, Haroche J, Charlotte F, et al: Erdheim-Chester disease in childhood: a challenging diagnosis and treatment. J Pediatr Hematol Oncol 31:782786, 2009

30. Veyssier-Belot C, Cacoub P, Caparros-Lefebvre D, Wechsler J, Brun B, Remy M, et al: Erdheim-Chester disease. Clinical and radiologic characteristics of 59 cases. Medicine (Baltimore) 75:157-169, 1996

31. Wilejto M, Abla O: Langerhans cell histiocytosis and Erdheim-Chester disease. Curr Opin Rheumatol 24:90-96, 2012

32. Wright RA, Hermann RC, Parisi JE: Neurological manifesta- tions of Erdheim-Chester disease. J Neurol Neurosurg Psychiatry 66:72-75, 1999

\section{Author Contributions}

Conception and design: Alimohamadi, Samii. Acquisition of data: Alimohamadi, Hartmann, Paterno. Analysis and interpretation of data: Alimohamadi, Paterno, Samii. Drafting the article: Alimohamadi, Hartmann. Critically revising the article: Alimohamadi, Paterno, Samii. Reviewed submitted version of manuscript: Alimohamadi, Hartmann, Samii. Approved the final version of the manuscript on behalf of all authors: Alimohamadi.

\section{Correspondence}

Maysam Alimohhamadi, Rudolf-Pichlmayr St., No. 4, Hannover 30655, Germany. email: alimohamadi59@gmail.com. 